\title{
Exploiting Capture Effect to Provide Service Differentiation in Wireless LANs
}

\author{
Alfandika Nyandoro* and Mahbub Hassan*† \\ ${ }^{*}$ School of Computer Science and Engineering \\ University of New South Wales \\ Kensington NSW 2052, Australia \\ Email:\{alfan,mahbub\}@cse.unsw.edu.au \\ $\dagger$ National ICT Australia Ltd \\ Locked Bag 9031 Alexandria NSW 1435 \\ Australia
}

\begin{abstract}
We investigate the use of transmit power as a mechanism for service differentiation in contention based wireless Local Area Network(LANs). We use a dual transmit power scheme where the power levels are specially selected to guarantee capture whenever a collision occurs between a single high power frame and one or more low power frames. We employ the Distributed Coordination Function (DCF) of the IEEE's 802.11 wireless LAN standard for our simulation experiments. The performance metrics considered are throughput and Medium Access Control (MAC) delay. We specifically investigate the level of differentiation achievable between high power and low power hosts given various ratios of high power to low power hosts. Our results show that differentiation is more distinct when the ratio of high power to low power hosts is small. It was also determined that in a cell with a fixed number of hosts capable of switching between the two power levels, there exists a threshold beyond which the MAC delay for the low power hosts actually decreases with increasing number of high power hosts.
\end{abstract}

\section{INTRODUCTION}

In contention based access networks, such as ALOHA [1] and its various derivatives, the channel is shared by a number of hosts. Since channel access is contention based, if two or more hosts attempt to send frames in the same time slot, a collision occurs resulting in the loss of all affected packets. However, if the colliding frames are such that one of the colliding frames' signal is sufficiently stronger than the aggregate strength of the rest, it is possible to correctly receive the former with the rest filtered out as noise. This is the so-called capture effect. Initial work on the capture effect (in the context of improving slotted ALOHA throughput) was first published by Metzner [2] more than two decades ago where it was observed that utilisation of ALOHA networks could be improved from $37 \%$ to $53 \%$ by simply dividing the transmitters into two groups, one group sending at high power and another sending at low power. Considerable work has been done on the capture effect since Metzner's pioneering research. Most of the existing works investigate the use of capture to enhance overall system throughput and fairness e.g [3], [4]. Others investigate the fundamentals of various capture models, e.g. for indoor and outdoor environments [5], [6] or with respect to the modulation and coding techniques used by the transmitters, e.g. [7], [8].
In recent times, interest on the effect of capture in IEEE 802.11 [9] based networks has grown [10]-[13]. In [11], the fairness of a wireless LAN in the presence of the capture effect was investigated. A new capture model was also proposed since the investigation concluded that existing capture models did not agree with empirical data. In [13], the capture probability in infrastructure wireless LANs was also investigated. In both [11] and [13], the contending hosts are of uniform transmit power; the difference in received signal strength (RSS) was due to the the near-far effect and multi-path propagation. In [12] the impact of heterogeneous transmit powers for hosts in an ad-hoc network was considered. The results indicated that the system suffered throughput degradation because high power hosts dominated the channel at the expense of low power hosts.

In this paper, we explore the possibility of achieving service differentiation by deliberately creating and exploiting the capture effect in contention based wireless LANs. We specifically consider the Distribution Coordination Function (DCF) of the IEEE's 802.11 standard. We note that although existing works acknowledge that capture results in unfairness on the channel, to our knowledge, none of the works have sought to leverage this unfairness in order to achieve service differentiation. Using transmit power for service differentiation appears attractive since no additional functionality is required at either the host or the access point. In addition, hosts are free to choose their transmit power, thus dovetailing well with the distributed nature of Carrier Sense Multiple Access with Collision Avoidance (CSMA/CA), the protocol on which DCF is based. Our simulation study considers both throughput and MAC delay in a wireless LAN where the hosts can switch between two power levels. The rest of this paper is organised as follows. In Section II, the system model guiding our simulation study is presented, followed by a discussion of the results in Section III. We conclude the paper in Section IV with some notes on our ongoing efforts in this area.

\section{SySTEM MODEL}

We simulate an ideal channel condition infrastructure wireless LAN in which hosts exchange frames through the Access 
Point (AP) which acts as the base station for all hosts in its service area (cell). All the hosts are dual power enabled, i.e. they can transmit at either of two power levels, $P 1$ and $P 2$, where $P 1$ is the high power and $P 2$ is low power. We therefore have two traffic classes in the cell, the high priority traffic transmitted at $P 1$ and the low priority (or best effort) traffic transmitted at $P 2$. To select the actual values for $P 1$ and $P 2$ we consider that if a frame arrives at a receiver with power $P_{h}$ in the same time slot as $n$ other frames of power $P_{i}$ where $1<=i<=n$, the frame with RSS $P_{h}$ can capture the receiver only if $P_{h}$ is sufficiently higher than the aggregate power of the $n$ interferers [14] i.e

$$
\frac{P_{h}}{\sum_{i=1}^{n} P_{i}}>z_{0}
$$

where $z_{0}$ is the capture ratio.It is well known that the $z_{0}$ for narrow band systems ranges between 1 and 10. For a Direct Sequence Spread Spectrum (DSSS) system such as is used in 802.11 wireless LANs, processing gain reduces the impact of interference signals such that the capture ratio $z_{0}$ can be even lower [15]. For our simulation we considered that the IEEE's 802.11 standard [9] specifies a minimum transmit power of $1 \mathrm{~mW}$ and a maximum of $1000 \mathrm{~mW}, 100 \mathrm{~mW}$ and $10 \mathrm{~mW}$ for USA, Europe and Japan respectively. Given this wide range, capture can be guaranteed by selecting $1000 \mathrm{~mW}$ and $1 \mathrm{~mW}$ for high and low power respectively. In a cell with a total of 40 hosts, capture can still occur even in the worst case when 1 high power host transmits in the same slot as 39 low power hosts. In this case

$$
\frac{P_{h}}{\sum_{i=1}^{n} P_{i}}>25,
$$

which is higher than the typical capture threshold for narrowband systems. We note that such an extreme choice of transmit power levels may not be necessary in real life systems since experimental results [10] indicate that capture occurs when the Signal to Noise Ratio (SNR) of the contending hosts differ by as little as $5 d B$. We further assume that the near-far effect has a negligible impact on capture probability. This arises from the fact only $P 1$ and $P 2$ values that maximise capture are selected. In addition, the very nature of wireless LANs assumes close proximity (tens of metres) between the hosts and the AP.

It is also assumed that the AP and the hosts have a line of sight (LOS) between them. This assumption is necessary to eliminate any differences in signal attenuation between different hosts and the AP that may result from obstacles in their paths. While the LOS assumption is not always valid, the trend of wireless LAN deployed in public spaces such as shopping malls, airport and hotel lobbies coupled with falling hardware costs suggest more dense AP deployment in future wireless LAN environments. Therefore that the presence of a LOS between mobile hosts and the AP is becoming more realistic.

In order to analyse the performance under heavy load, we assume that each host always has a frame awaiting transmission immediately after each successful transmission. We limit the total number of hosts that can be associated with an AP
TABLE I

SimUlation PARAMETERS

\begin{tabular}{|l|l|}
\hline Data Payload & 1023 bytes \\
Channel Bit Rate & $1 \mathrm{Mbps}$ \\
Acknowledgement (ACK) size & $10 \mu \mathrm{s}$ \\
Medium Access Control (MAC) header size & 30 bytes \\
Short Inter-frame Frame Space (SIFS) duration & $14 \mu \mathrm{s}$ \\
slot-time & $20 \mu \mathrm{s}$ \\
DCF Inter-frame Space (DIFS) & $50 \mu \mathrm{s}$ \\
ACK-timeout & $250 \mu \mathrm{s}$ \\
propagation delay & $1 \mu \mathrm{s}$ \\
Min Contention Window Size(W) & 32 \\
Maximum Back-off Stage (m) & 5 \\
& \\
\hline
\end{tabular}

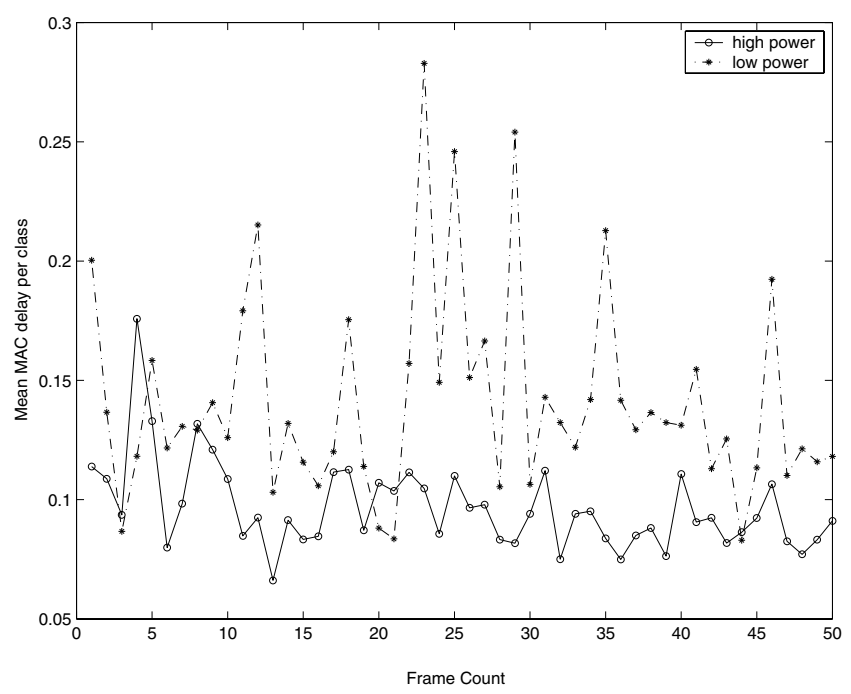

Fig. 1. Delay per class for $1: 1$ ratio of $P 1$ to $P 2$ hosts

at any one time to 40 because realistically, it is unlikely that more than 40 users would gather in the limited area that an AP services. The wireless LAN considered conforms to the IEEE's 802.11 with parameters shown in Table I. We considered only $1 \mathrm{Mbps}$ since it is the basic rate of the wireless LAN standard.

\section{RESULTS}

The investigation we carried out consisted of a number of scenarios as discussed below. Incorporated in the delay results are error bars representing the standard deviation from the mean. The throughput results do not have significant variations, hence the error bars were omitted there.

\section{A. Scenario I}

In the first scenario, we consider a cell with a total of 10 hosts divided equally between high priority transmitters and low priority transmitters. Figure 1 plots the mean frame-byframe MAC delay of each class for the first 50 frames. It can be seen that the 5 high power hosts achieve a mean MAC delay of around $0.1 \mathrm{~s}$ compared to about $0.15 \mathrm{~s}$ for low power hosts, i.e. on average low power hosts experience 1.5 times the delay experienced by the high power hosts. 


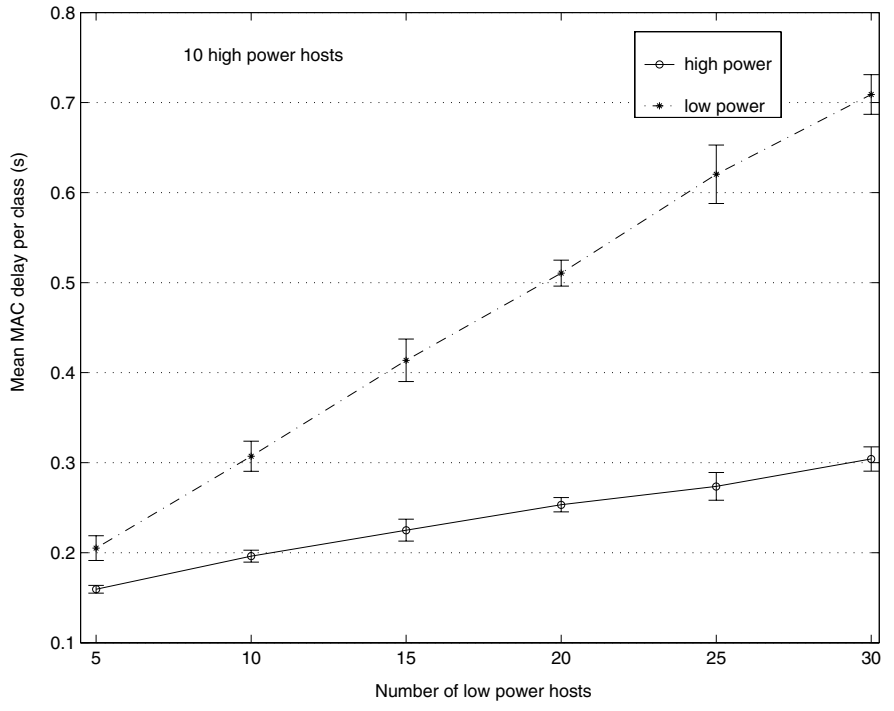

Fig. 2. Delay for a fixed number $P 1$ hosts as number of $P 2$ hosts increasees

\section{B. Scenario II}

In the second scenario, we investigate what happens when the number of high priority hosts is fixed at 10 while the number of best effort hosts is allowed to increase, up to the combined upper limit of 40 hosts in the cell. The effect on MAC delay is shown in Figure 2. It can be seen that the delay for both classes increases linearly with increasing number of low power hosts. As in Figure 1, when the ratio of high priority to low priority hosts is $1: 1$, i.e. 10 low power hosts and 10 high power hosts, the delay ratio (low power : high power) is around 1.5. As more best effort hosts join the cell, the average delay for high priority hosts increases from an initial value of $0.16 s$ when there are only 5 best effort hosts in the cell to $0.3 \mathrm{~s}$ when their number increases to 30 . On the other hand, the average delay for the best effort class more than trebles from $0.2 \mathrm{~s}$ at the beginning to more than $0.7 \mathrm{~s}$ when their number increases to 30 . From the foregoing, an important observation can be made; high priority traffic enjoys relative protection from the impact of increasing low power traffic. Given an long term upper limit on the priority traffic delay, it is permissible to allow allow many best effort host to join the cell without a negative impact on the priority traffic.

Conversely, as discussed later in Scenario III, the number of high priority hosts has to be kept within firm limits to maintain performance bounds.

Figures 3 and 4 show the throughput results obtained under Scenario II. In Figure 3 the overall throughput achieved by hosts in each class is shown. It can be seen that the high power and low power hosts achieve equal throughput when the ratio of low to high power hosts is 2 . This implies that the high power hosts achieve twice as much throughout as the low power hosts in this combination. Figure 4 shows the average per host throughput in each class. It can be seen that the throughput achieved by low power hosts decreases more rapidly compared to that for high power hosts, as a result

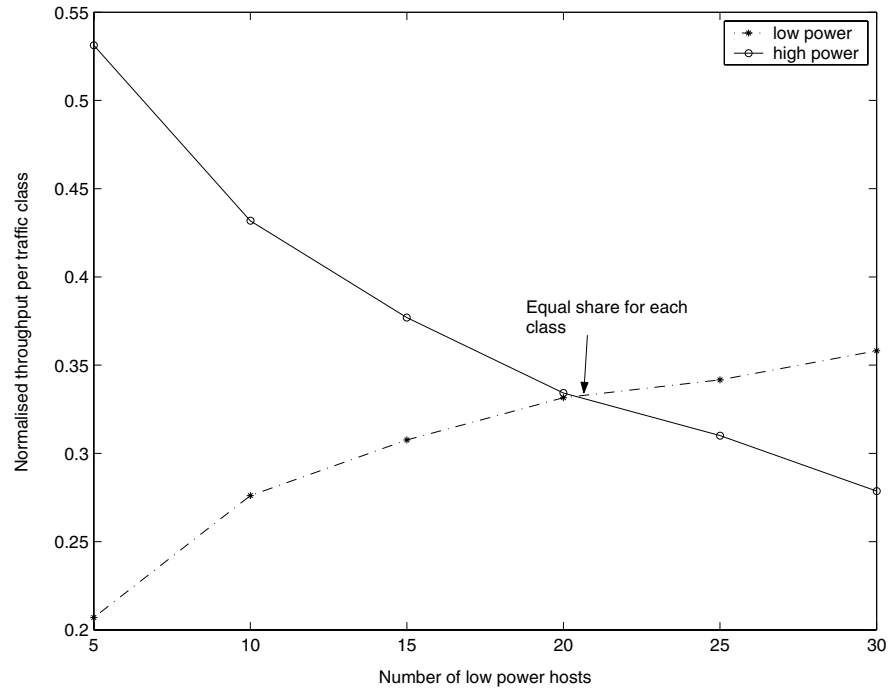

Fig. 3. Throughput per class as number of $P 2$ hosts increases

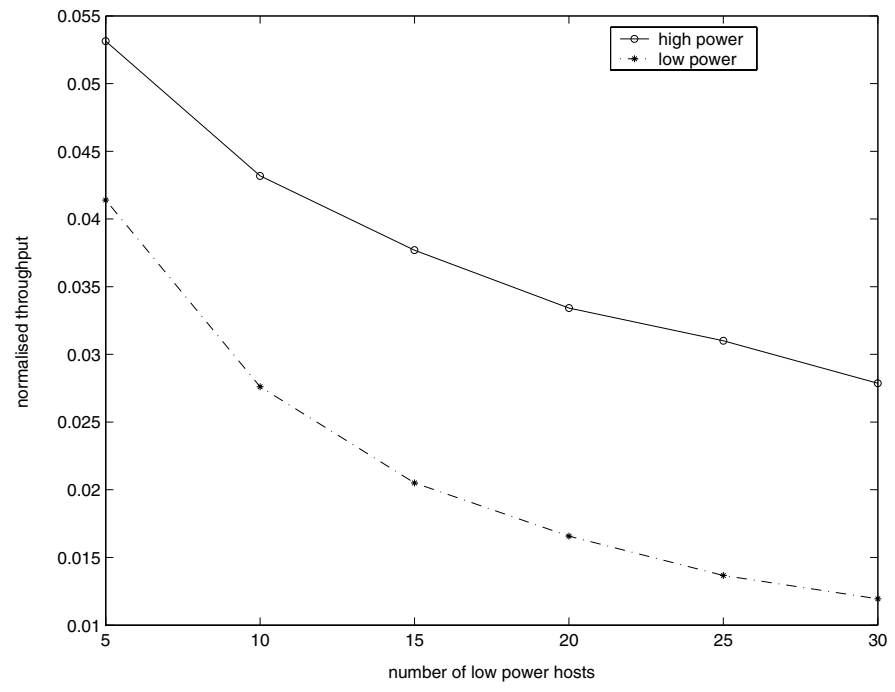

Fig. 4. Mean individual delay as number of $P 2$ hosts increases

the throughput difference increases with number of low power hosts.

\section{Scenario III}

This scenario investigates the situation where the total number of hosts in the wireless cell is fixed at 40 hosts, but the hosts switch their transmission power between high and low. As the number of high priority hosts increases, the number of low priority hosts decreases and vice versa. Figure 5 shows the mean delay for each traffic class when the number of high priority hosts increases as shown on the x-axis. Initially, there are very few high priority hosts so the cell is dominated by best effort hosts. As a result, high priority hosts enjoy much lower delay. As more hosts start to switch to $P 1$, the delay for high power hosts increases because the probability of the high power hosts colliding amongst themselves increases. On the other hand, the mean delay for the low power hosts also 


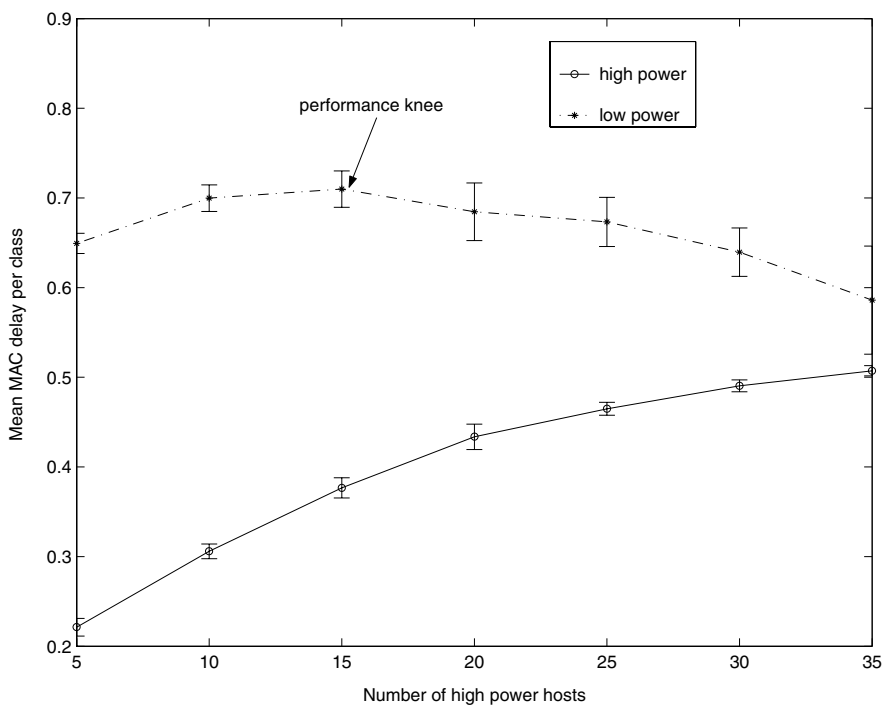

Fig. 5. Mean delay per host as number of $P 1$ hosts increases

increases up to a point. The increase is due to the advantage that the high power hosts have at the expense of low power. As more and more hosts switch to high power, the delay advantage that high power hosts enjoy over low power begins to diminish. This occurs because with an increasing number of high power hosts comes the higher collision probability amongst hosts in the same class resulting in a larger mean contention window size for the class. The combination of a decrease in number of low power hosts and an increasing mean contention window size for high priority results in the performance knee shown in Figure 5.

When most hosts switch to high power, no significant performance is achieved by the high power hosts. At the extreme end when all hosts switch to high power, the performance advantage vanishes as the system reverts to the standard single power level. Clearly sending at high power for no performance gain is undesirable since the battery drains more quickly so hosts may revert to the standard transmit power. This performance dynamics may lead to interesting cycles, where users first start to switch to high power and continue to do so until they notice no significant performance gain, and then start to decrease their power level. At a later time, the users may observe that switching to a higher power again provides better performance, however other users may also behave likewise, thus perpetuating the power switching cycles.

In Figure 6 we show the throughput achieved by each transmit power class as the number of high power hosts increases from 5 to 35 . As seen in previous scenario results, the throughput for the high priority class equals that of the best effort class when the ratio of low to high hosts is 2 . However what is more interesting is the differentiation achieved on per host basis. Figure 7, shows the per host throughput with increasing number of high power hosts. At the beginning, each of the 5 transmitting at $P 1$ achieves an average throughput of

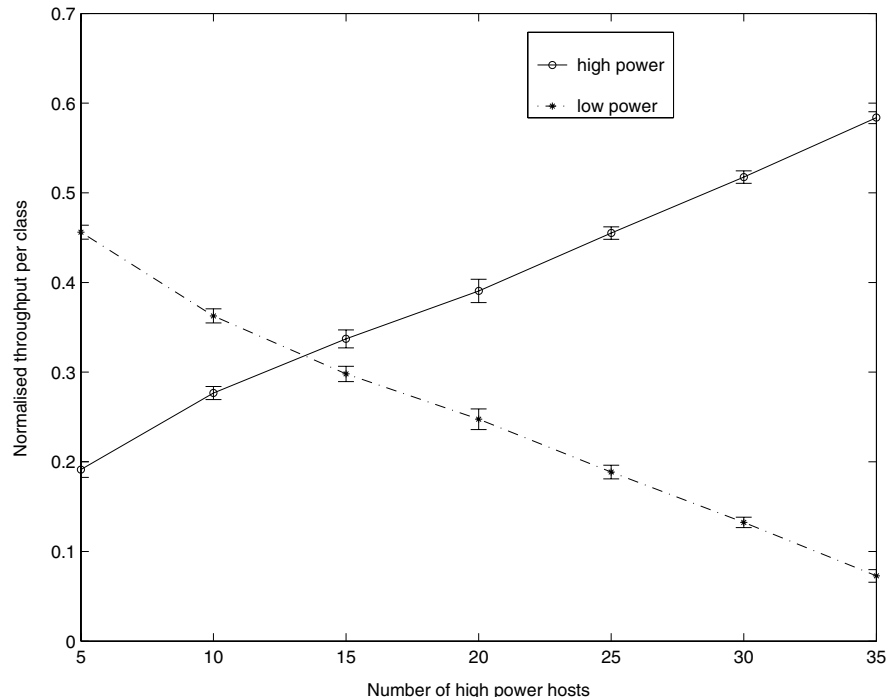

Fig. 6. Class throughput as ratio of $P 1$ to $P 2$ hosts increases

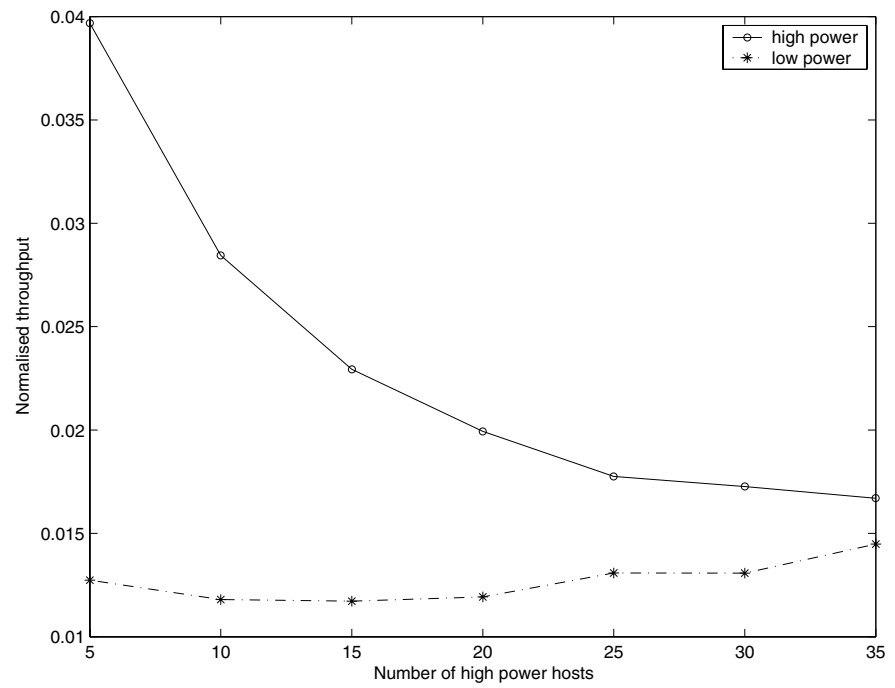

Fig. 7. Mean per host throughput as ration of $P 1$ to $P 2$ increases

0.04 , i.e. $40 \mathrm{Kbps}$ each compared to 0.0125 ; about $(12.5 \mathrm{Kbps})$ for low power. When high priority hosts dominate the cell, the throughput of low priority hosts rises, but only marginally. As discussed under delay performance above, this is due to a slight increase in transmission opportunities due to high priority hosts colliding amongst themselves. At the extreme end when all the hosts switch to high power, the performance advantage for the priority class vanishes as can be seen by the converging throughput plots.

The impact of the population of high power hosts on both throughput and delay is more pronounced than that of low power. Accordingly, if there are performance bounds to be maintained, it is the number of high power hosts that has to be kept in check. The impact of low power hosts is small as shown in Scenario II. 


\section{CONCLUSION}

We have shown some quantitative results of a dual transmit power service differentiation scheme in contention based wireless LANs. The service differentiation is in terms of both delay and throughput. In a system where the hosts can independently switch transmission power as per their quality of service (QoS) needs, there is potential to create transmit power cycles as hosts switch from low power to high power so as to gain a service advantage and back again to low power when too many hosts move to the high power such that performance diminishes. In addition, there exists a performance knee where the delay of low power begins to fall with increasing number of high power hosts. Since our current results are based on simulations of a wireless cell we are presently developing a Markov chain model to analytically predict the performance dynamics for multiple power level scenarios. This model will help in deriving performance bounds for different traffic mixes.

\section{REFERENCES}

[1] N. Abramson, "The ALOHA system - another alternative for computer communications," in Proc. Fall Joint Comput. Conf., AFIPS Conf., vol. 37, 1970, pp. 281-285.

[2] J. Metzner, "On improving utilisation in ALOHA systems," IEEE Trans. Commun., vol. 24, pp. 447-448, Apr. 1976.

[3] J. H. Sarker, M. Hassan, and S. J. Halme, "Power level selection schemes to improve throughput and stability of slotted ALOHA under heavy load," Computer Communications, vol. 25, pp. 1719-1726, 2002.

[4] V. Wong and C. Leung, "A transmit power control scheme for improving performance in a mobile packet radio system," IEEE Trans. Veh. Technol., vol. 43, pp. 174-180, February 1994.

[5] J. Sanchez-Garcia and D. R. Smith, "Capture probability in Rician fading channels with power control in the transmitters," IEEE Trans. Commun., vol. 50, pp. 1889-1891, Dec. 2002.

[6] J.-P. M. Linnartz, R. Hekmat, and R.-J. Venema, "Near-far effects in land mobile access networks with narrow-band rayleigh fading channnels," IEEE Trans. Veh. Technol., vol. 41, no. 1, pp. 77-90, 1992.

[7] M. Soroushnejad and E. Geraniotis, "Probability of capture and rejection of primary multiple-access interference in spread-spectrum networks," IEEE Trans. Commun., vol. 39, pp. 986-994, June 1991.

[8] W. Ren, J. Ward, S. Hodgart, and M. Sweeting, "Capture effect and modelling for coherent BPSK," IEE Electonics Letters, vol. 35, pp. 2079-2080, 1999.

[9] Wireless LAN Medium Access Control (MAC) and Physical Layer (PHY) Specification, IEEE Std. 802.11, 1999.

[10] C. Ware, J. Judge, and E. Dutkiewcz, "Unfairness and capture behaviour in 802.11 adhoc networks," in Proc. IEEE International Conference on Communications ICC'00, vol. 1, June 2000, pp. 159-163.

[11] C. Ware, J. Chicharo, and T. Wysocki, "Simulation of capture behaviour in IEEE 802.11 radio modems," in Proc. IEEE Vehicular Technology Conference (VTC 2001 Fall), Oct. 7-11, 2001, pp. 1393-1397.

[12] N. Poojary, S. V. Krishnamurthy, and S. Dao, "Medium access control in a network of Ad Hoc mobile nodes with heterogeneous power capabilities," in Proc. IEEE 5th International Conference on Communications, vol. 3, June 11-14, 2001, pp. 872-877.

[13] J. Y. H. Zhao and O. C. Ugweje, "Analysis of capture probability performance techniques for wireless LAN," in Proc. IEEE Vehicular Technology Conference (VTC 2002 Spring), vol. 3, May 6-9, 2002, pp. 1190-1194.

[14] J. C. Arnbak and W. van Blitterswijk, "Capacity of slotted ALOHA in Rayleigh Fading Channels," IEEE J. Select. Areas Commun., vol. SAC5, no. 2, pp. 261-269, February 1987.

[15] B. Hajek, A. Krishna, and R. O. Lamaire, "On the capture probability for a large number of stations," IEEE Trans. Commun., vol. 45, no. 2, pp. 254-260, Feb. 1997. 